\title{
A Vocal Based Handheld Assister For Prosopagnosics
}

\author{
${ }^{*}$ Athira $S^{1}$, Dhanya $V^{2}$, Sreeja $S^{3}$, Chandni C $S^{4}$ \\ Department of Electronics and Communication, \\ Prime College of Engineering, Palakkad, Kerala, India \\ Corresponding Author: Athira S
}

\begin{abstract}
The proposed system is a real time low cost portable computerized face perception system which acts as an assister for Prosopagnosics in their social lives who are suffering from the inability to recognize or identify their near ones. The system prescribed here is standalone, capable of doing both face detection and face recognition. The device comes with an RFID receiver and camera attached to the raspberry pi worn by the patient. Raspberry-Pi forms the heart of the system and uses Linux OS with the Python language for the software part. The human face is detected using Haar features and Eigen face algorithm with its low dimensionality is used for face recognition.The identity of the person is informed vocally to the patient via an earphone.
\end{abstract}

Keywords: Face perception, Prosopagnosics, RFID, Raspberry Pi, Eigen face, Haar algorithm, Python.

\section{Introduction}

Prosopagnosia is characterized by a person's inability to recognize faces. People with Prosopagnosia, referred to as Prosopagnosics, find it difficult to distinguish facial features and differentiate people in their social lives. The most prevailing strategy for prosopagnosics is to learn to identify people by their clothing, gait, and other distinctive features. However, memorizing various details puts heavy burden on the patients. Since patients' visual processing and intellectual functioning remain intact, the assistance provided by our system to compensate their inability in face recognition can help them in living a normal life.

The system comes with two level of operation, i.e., detection of a human face from an image and also recognition of the person. The two operations are combined together into a single standalone and portable system. The system is standalone in the sense that both training and recognition is carried out without the help from any additional systems like computers. The system has an RFID reader that reads data from the RFID tag given to the trained persons. The camera attached to the Raspberry Pi captures the image of the person standing in front of the patient and the image processing is under taken by the Raspberry-Pi. Thus, the camera used here is a low power Omni-Vision CMOS OV5647 camera. The Raspberry-Pi is a single on- board computer which comes with a Micro-SD card for data storage, necessary during imagecomparison using Eigen face algorithm, which provides lower dimensionality and its computational speed for face recognition is very high. The Haar algorithm is used for face detection.

Raspberry-Pi chooses Linux and Python as its principle operating system and programming language respectively. The system also provides the advantage of low cost and wear-ability. When the face is recognized, the information of the recognized person, which is stored during training, is produced vocally and is sent to an earphone. The same information will be displayed on the LCD.

\section{History}

In this section, a set of systems that have similar design goals as that of the proposed system is discussed. According to [8], Lienhart.R and Moyelt.J developed a system in 2002 which is based on a set of Haar like features for object detection. But this system is more complex since it involves more number of feature extension. Moreover, it requires high resolution camera. In 2006, Stanz.Li and lun zhang developed a near infrared (NIR) image based face recognition system as mentioned in [12]. This design minimizes the influence of environmental lighting on face images. This design has a drawback of poor matching scheme and the complete co-operation of the user is important. Attendance Management System (AMS) is the easiest way to keep track of attendance The automated attendance management system in [6] with the use of Raspberry Pi along with RFID gives a base to the proposed system. Farajzadeh,N.Farz.k described a hybrid face detection system as in [5] which is based on Viola and Jonespsilas work and uses Radial Basis Neural Network (RBFNN).The drawback of this system is that it is more complex. and is highly expensive. Kequing Shi,Shurong Pang and Fengqi Yu developed a real time face detection and recognition system. This system's performance depends on Haar features. Major drawbacks of this design are high dimensionality, complexity and 
cost. The lastly described work is closely related to the proposed system. The system that we are describing through this paper has the capability of detecting and recognizing the faces at a time. Moreover, it is simple, portable and low cost.

\section{Block Diagram}

The system mainly consists of Raspberry Pi, 5MP Omni Vision camera, 16×2 LCD. Raspberry Pi is the heart of the system. It uses a credit card sized computer. Fig. 1 shows the block diagram. It includes an ARM11 700 MHz processor, video core, 256 megabytes of RAM. An RFID reader module

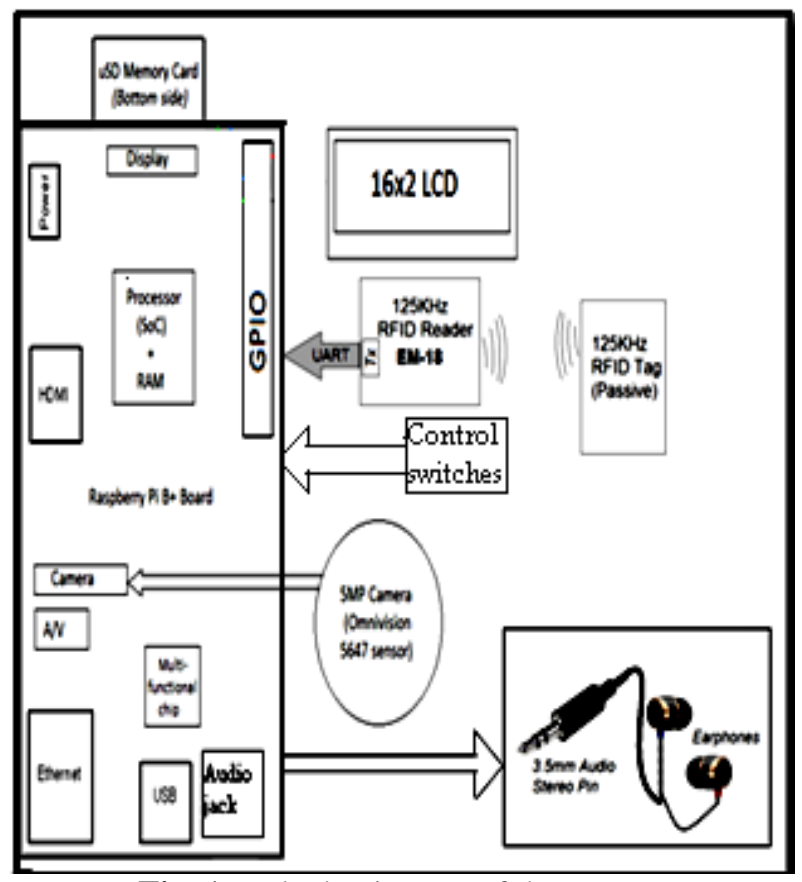

Fig. 1. Block Diagram of the system

is interfaced with the Raspberry Pi GPIO via UART. EM-18 RFID reader module is used and can read any $125 \mathrm{KHz}$ tags. It features low cost, low power consumption and provides UART output format. Raspberry Pi allows for high definition video streaming and other basic computer functions like word processing and web browsing. It makes the system portable, easy to use and real time. It is versatile and powerful and stores the data on micro- SD card. It provides a general purpose remote I/O expansion for most microcontroller families via the two line bidirectional bus. The control switches are used as inputs to the Pi module to initiate the training and recognition. Thus entire control of the system is with the user, these control switches providing communication between the patient and the system. The Omni Vision 5647 CMOS sensor camera module is a custom designed add on for Raspberry Pi. It is attached to the Raspberry Pi by one of the two sockets on the upper surface of the board via a short ribbon cable. The interface uses a dedicated camera serial interface which has designed especially for interfacing camera. The mounted camera captures the image of the person standing in front of the patient and sends it to the BCM2835 processor in the Raspberry Pi, where it process the image using face recognition and detection algorithm to detect and recognize the face in each frame. After processing, the information is passed to LCD which displays whether the person standing in front is known or unknown to the patient. The information of the recognized person is conveyed vocally to the user via earphones through the audio jack output of the Raspberry Pi Python is a powerful programming language that is easy to use with Raspberry Pi. Its elegant syntax allows clear definition application behavior using fewer lines of codes than that would be required in any other languages. It supports multiple programming paradigms including comparative, functional and object oriented styles, allowing a wide range of task to be performed.

\section{System Description}

The system plays dual role: face detection and recognition using appearance based approaches. The models are learned from a set of training images which capture the representative variability of facial appearance. Eigen face algorithm helps the face recognition to be achieved real time once Eigen faces of the database are calculated and stored in the MMC card.The persons are trained initially by capturing a number of images using Pi camera. The captured images are then converted into gray scale images by applying Eigen face 
algorithm. The information regarding the trained person is also stored along the images. The RFID reader provided with the patient compares and matches the RFID tag given to the trained person and finally face recognition is employed. The output of the face recognition carried out by the raspberry pi module is displayed on the LCD and at the same time produces a vocal output via ear phone.

\section{A. Eigen Face Generation and Practical Implementation}

Eigen face algorithm helps the face recognition to be achieved real time once Eigen faces of the database are calculated. Eigen faces is the name given to a set of Eigen vectors when they are used in the computer based systems. The Eigen vectors are derived from the covariance matrix of the probability distribution over the high dimensional vector space of face images. This produces dimension reduction by allowing the smaller set of basis images to represent the original training images. To recognize faces, gallery images, those seen by the system, are saved as collections of weights describing the contribution each Eigen face has to that image. When a new face is presented to the system for classification, its own weights are found by projecting the image onto the collection of Eigen faces. This provides a set of weights describing the probe face. These weights are then classified against all weights in the gallery set to find the closest match.

OpenCV contains a set of negative images and those images being captured for training are saved as positives. The training includes generation of positive and negative Eigen face images from the mean face and captured positives. This positive Eigen faces are a summary of the features that differentiate the particular image from the mean face. To be recognized, captured face must be a positive prediction below positive-threshold configuration value.

\section{B. Haar Algorithm}

A simple rectangular Haar-like feature can be defined as the difference of the sum of pixels of areas inside the rectangle, which can be at any position and scale within the original image. This modified feature set is called 2-rectangle feature. The values indicate certain characteristics of a particular area of the image. Each feature type can indicate the existence (or absence) of certain characteristics in the image, such as edges or changes in texture. A window of the target size is moved over the input image, and for each subsection of the image the Haar-like feature is calculated. This difference is then compared to a learned threshold that separates non-objects from objects. Because such a Haar-like feature is only a weak learner or classifier a large number of Haar-like features are necessary to describe an object with sufficient accuracy.

\section{Software}

The Raspberry Pi primarily uses Linux kernel based operating systems. The Operating System that is used is NOOBS with python for both training and recognizing part of the system as it is best suited for Raspberry Pi.. It's a derivative of the Linux OS. Specific applications of this software include added multimedia functionalities (essential libraries and packages to our project). It offers a Graphic User Interface which is intuitive to use along with the regular Linux command line interface. To install this, the image files of Raspbian OS are downloaded from the Raspberry Pi forum. This image file is extracted and is subsequently burned to the $\mathrm{SD}$ card using the software SD Formatter. The SD card is inserted to the Pi and it is given power supply. Default configuration settings are set.

The ARM 11 chip at the heart of the Pi is based on version 6 of the ARM. Thus, it is not possible to run windows or latest version of Linux on Pi. Similarly, the Pi has adopted python as its principle user programming language. The python is interpreted as graphical user interface. Python gains more concentration as it is able to get work done with fewer lines of code and high standards of quality with coding, testing and documentation standards. The system depends on the Open CV computer vision library to perform face detection and recognition.

\section{RFID Interfacing With Raspberry Pi}

RFID (Radio Frequency Identification) uses electromagnetic fields to read, monitor and transfer data from tags attached to different objects. It is not necessary that the cards are to be in visibility of the reader, it can be embedded in the tracked object. The tags can be actively powered from a power source or can be passively powered form the incoming electromagnetic fields. EM-18 RFID reader module is used here and can read any $125 \mathrm{KHz}$ tags. It features low cost, low power consumption, and easy to use. It can be directly interfaced with Raspberry Pi using UART and with PC using an RS232 converter. The RFID tag is used as an identity for a particular user. If the identity (serial number of the tag) of the user is matched with the one already stored in this system, he gets immediate access through it. In beginning, the user is prompted to scan his tag or ID. 


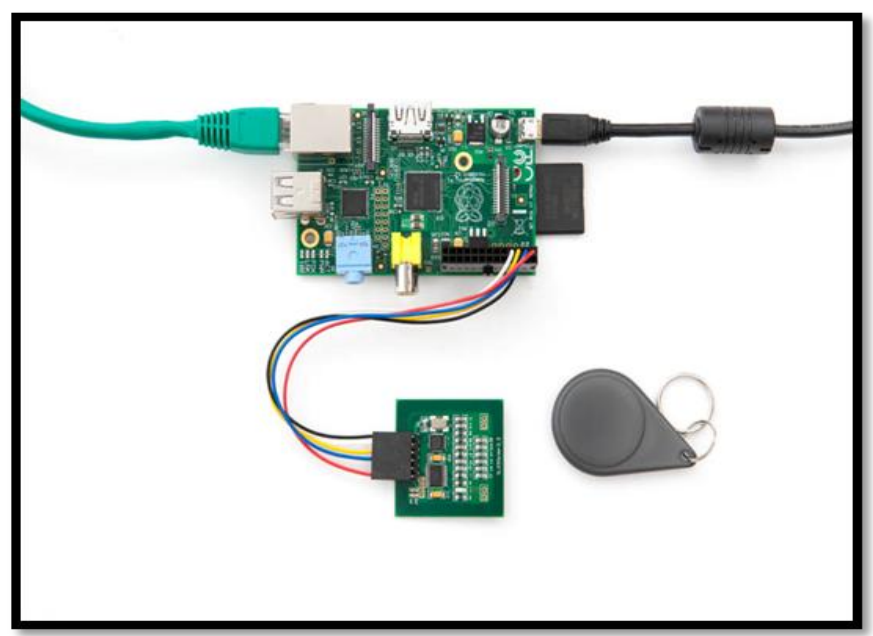

Fig. 2 RFID and Raspberry Pi Interface

The serial code of the tag is identified by the reader module and is sent to Raspberry Pi for checking. If the ID is matched by the microcontroller, the user gets the access. On the contrary, if the tag is not identified, a message ('Wrong ID') is displayed on LCD screen.

\section{Process Flow}

The process involves the following steps and is diagrammatically shown by a flow chart in Fig 3 .

1. Start

2. Initialize camera, LCD, RFID reader and earphones.

3. Check whether the RFID reader reads valid data from the RFID tag.

4. If yes, face recognition starts with a beep. The Pi camera attached to the Raspberry Pi captures the image of the RFID tag bearing person. Then the system compares the captured image with trained images and displays the information on the LCD. The earphones produce the same vocally. The process the moves to step 8.

5. If no, LCD displays "unknown person" and the earphones produces the result vocally.

6. The system then checks whether any training is needed.

7. If yes, image acquisition is employed. Then Eigen face algorithm is computed to obtain gray scale images which are stored along with the name and relation of the trained person. If no, process moves to step 8 .

8. Stop.

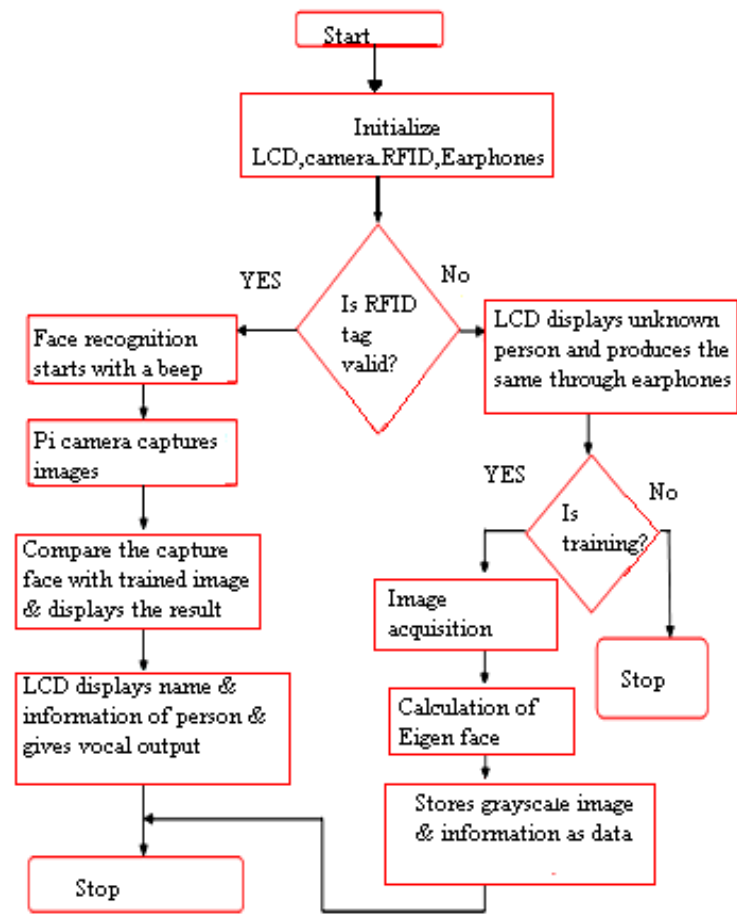

Fig. 3 Process flow of the system 


\section{Merits And Demerits}

System developed in this paper is useful for real time applications, which can perform both face recognition and detection of human faces. Raspberry Pi model is used which makes the system portable, stand alone and low cost. Moreover it consumes less power which leads to increased performance. Large variation in visual stimulus affects due to the viewing conditions, aging, expression etc. Hence $100 \%$ accuracy is not promisable. The RFID reader must be provided to each trained person.

\section{Results And Discussion}

As the part of our work, we conducted face recognition and detection of 40 test images under varying parameters. At first, 40 test images were captured using Raspberry Pi camera module and were trained. Recognition of people were done in two different environment; one in normal situations with improper lighting and other with proper fixed lighting. On our analysis we found that, RFID tags of 39 people were valid. Out of these valid tags, under improper lighting conditions about 32 images were recognized. Under proper lighting, recognized images were improved from 32 to 37 images. Ignoring the artificial environment and considering only the normal condition we found out the efficiency of our system is nearly $80 \%$; results obtained during different lightning conditions are plotted in fig 4 .

\section{Conclusion And Future Scope}

The system designed acts as an interface for the prosopagnosics to identify different faces which is the primary factor in social interactions and allows them to behave normally in social lives. This system is very small and portable making it possible for patients to wear it during their daily activities and hence it is made stand alone and operate in a low power. This work can be utilized for wide range of image processing applications, security applications, robotics, embedded systems, human computer interactions etc.

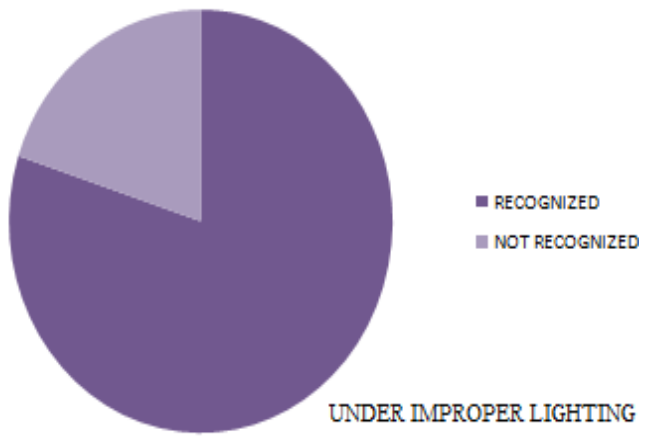

(a)

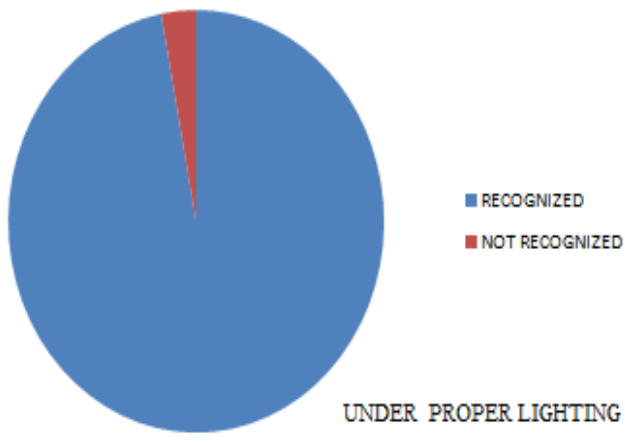

(b)

Fig. 4 Accuracy of the system under (a) improper lighting condition (b) proper lighting condition

The system is also useful for handicapped and old age ones in improving their social lives In conclusion; the paper offers a system that has a wide developmental area, both in research and commercial fields, i.e., possibility of enhancing the system is endless. Authenticity can be increased by including iris recognition which also improves its accuracy. All the modifications can be integrated to the system with less effort. In future; voice recognition can be incorporated to the system to extend its application level and accuracy. Also wireless camera can be installed to make the users more comfortable and reduces system complexity. 


\section{Acknowledgment}

The authors are grateful for the assistance provided by Department of ECE of Prime college of Engineering, Palakkad.

\section{References}

[1] A.K.M Fazla Mehrab, Palash Debnath, G.M. Mashrur-E-Elahi "An Approach to Real-Time Portable Device for Face Recognition System" $15^{\text {th }}$ International Conference on Computer and Information Technology (ICCIT), ISBN: 978-1-4673-4834-8, pp. 126-131, December 22-24, 2012

[2] Aditya Nigam, Phalguni Gupta, “A New Distance Measure for Face Recognition System"2009 Fifth International Conference on Image and Graphics.

[3] Andrew Wagner, John Wright, Arvind Ganesh, Zihan Zhou, Hossein Mobahi, and Yi Ma. IEEE Transactions on Pattern Analysis and Machine Intelligence (PAMI), vol. 34, no. 2, February 2012.

[4] BeumerG.N, Tao.Q, A.M. Bazen, and Veldhuis R.N.J, “A landmark paper in face recognition"7-th IEEE International conference on Automatic Face and gesture recognition 2006.

[5] Farajzadeh, N. Faez, K. "Hybrid face detection system with robust face and non-face discriminability" Published in Image and Vision Computing New Zealand, 2008. IVCNZ 2008. 23rd International Conference IEEE 2008 PAGES 1-6.

[6] G.Lakshmi Priya, M.Pandimadevi , G.Ramu Priya , P.Ramya , “ Implementation of Attendance Management System using SMART-FR", International Journal of Advanced Research in Computer and Communication Engineering, Vol. 3, Issue 11, November 2014

[7] Johannes Stallkamp, Hazim K. Ekenel, Rainer Stiefelhagen, "Video-based Face Recognition on Real-World Data," 11th IEEE International Conference on Computer Vision, 2007, pp:1-8.

[8] Lienhart, R, Maydt, J. "An extended set of Haar-like features for rapid object detection",Proceedings of 2002 International Conference on Image Processing. (Volume:1) Page(s) I-900 - I-903 vol.1

[9] Lijun YinJohnny Loi Wei Xiong, "Facial Expression Representation and Recognition Based on Texture Augmentation and Topographic Masking", IEEE Proceedings of the 12th ACM International Conference on Multimedia, New York, NY, USA, October 10-16, 2004.

[10] Pankaj R.Bhusari, Vrushali G.Raut, "Real Time Face Detection and Recognition System ,IEEE 2012

[11] Sima Taheri, Vishal M. Patel, and Rama Chellappa, "Component-based Recognition of Faces and Facial Expressions",IEEE Transactions on Affective Computing, 2013.

[12] Stan Z. Li, Lun Zhang, Sheng Cai Liao, Xiang Xin Zhu, Ru Feng Chu, Meng Ao, Ran He "Near-infrared Image Based Face Recognition System", -7- th IEEE International Conference on Automatic Face \& Gesture Recognition,2006.

[13] V. Sathish Kumar ,G.Senthilkumar1, K.Gopalakrishnan2 , "Embedded image capturing system using raspberry pi system", International Journal of Emerging Trends \& Technology in Computer Science (IJETTCS), Volume 3, Issue 2, March - April 2014.

[14] William Robson Schwartz, , Huimin Guo, Jonghyun Cho, and Larry S. Davis, "Face Identification Using Large Feature Sets" IEEE Transactions on Image Processing, Vol. 21, no. 4, April 2012.

[15] Xi Wang, Xi Zhao, Varun Prakash, Weidong Shi, and Omprakash Gnawali, "Computerized-Eyewear Based Face Recognition System for Improving Social Lives of Prosopagnosics” ,Proceedings of the 7th International Conference on Pervasive Computing Technologies for Healthcare May 2013

[16] Yao-Jiunn Chen, Yen-Chun Lin, "Simple Face-detection Algorithm Based on Minimum Facial Features", The 33rd Annual Conference of the IEEE Industrial Electronics Society (IECON) Nov. 5-8, 2007, Taipei, Taiwan.

IOSR Journal of Electronics and Communication Engineering (IOSR-JECE) is UGC approved Journal with Sl. No. 5016, Journal no. 49082.

Athira S. "A Vocal Based Handheld Assister For Prosopagnosics." IOSR Journal of Electronics and Communication Engineering (IOSR-JECE) 12.4 (2017): 40-45. 